\title{
Acknowledgment to reviewers
}

Joshua Akinyemi, University of Ibadan (Nigeria)

Roderic Beaujot, Western University (formerly University of

Western Ontario), London (Canada)

David E. Bloom, Harvard University, Cambridge, MA (USA)

Christine Bycroft, Statistics New Zealand, Wellington

James Frankish, University of British Columbia, Victoria (Canada)

Patrick Gerland, United Nations Population Division, New York

Robert Murdie, York University, Toronto

László Németh, Max Planck Institute for Demographic Research, Rostock (Germany)

Bali Ram, Western University (formerly University of Western

Ontario), London (Canada)

K.V. Rao, India Network Foundation, Orlando (USA)

Ana Fostik Sanchez, McGill University, Montreal

Martin Spielauer, Statistics Canada, Ottawa

Chirayath Suchindran, University of North Carolina at Chapel Hill (USA)

Zongli Tang, Auburn University at Montgomery (USA)

Rosemary Venne, University of Saskatchewan, Saskatoon (Canada) 